\title{
The MICE experiment PID instrumentation system
}

\author{
M. Bonesini* \\ sezione INFN Milano Bicocca, Piazza Scienza 3, Milano, Italy \\ E-mail: maurizio.bonesini@mib.infn.it
}

\begin{abstract}
The international Muon Ionization Cooling Experiment (MICE) will perform a systematic investigation of ionization cooling of a muon beam. The demonstration comprises one cell of the neutrino factory cooling channel. As the emittance measurement will be done on a particle-byparticle basis, sophisticated beam instrumentation is needed to measure particle coordinates and timing vs RF phase. A PID system, in order to keep beam contamination $(e, \pi)$ well below $1 \%$, based on time-of-flight stations, two Aerogel Cerenkov detectors and a KLOE-like calorimeter has been constructed and installed at RAL. It will be soon complemented by a fully active calorimeter (EMR) to measure precisely the muon range. Performances in beam at RAL are compatible with requirements and give the possibility to characterize fully the incoming muon beam (Step I of the MICE experimental program).
\end{abstract}

35th International Conference of High Energy Physics - ICHEP2010,

July 22-28, 2010

Paris France

*Speaker.

${ }^{\dagger}$ on behalf of the MICE Collaboration. 
The MICE experiment [1] at RAL aims at a systematic study of a section of the cooling channel of the Feasibility Study 2 of a Neutrino Factory [2], attaining a $10 \%$ effect for a $6 \pi \mathrm{mm} \cdot \mathrm{rad}$ impinging $\mu$ beam. The $5.5 \mathrm{~m}$ long cooling section consists of three liquid hydrogen absorbers and eight $201 \mathrm{MHz}$ RF cavities encircled by lattice solenoids (see figure 1). As conventional emittance measurement techniques reach barely a $\sim 10 \%$ precision, a novel method based on single particle measurements has been envisaged. Particle coordinates are measured before and after the cooling section by two magnetic spectrometers (x,y,z, $\left.p_{x}, p_{y}, p_{z}\right)$ complemented by TOF detectors (t), obtaining a $0.1 \%$ precision on the determination of the input and output emittances, thus allowing a sensible extrapolation to the full cooling channel of a Neutrino Factory.

Particle identification (PID) is obtained upstream of the first tracking solenoid by two TOF stations (TOF0/TOF1) (below $210 \mathrm{MeV} / \mathrm{c}$ ) and two threshold Cerenkov counters (CKOVa/CKOVb), that will provide $\pi / \mu$ separation between 210 and $365 \mathrm{MeV} / \mathrm{c}$. Downstream the PID is obtained via a further TOF station (TOF2) and calorimeters (EMCAL), to separate muons from decay electrons.

To determine the timing with respect to the RF phase to a precision of $5^{\circ}$ a detector resolution better than $70 \mathrm{ps}$ is needed for TOF0. To allow a better than $99 \%$ rejection of pions in the incoming $\mu$ beam, a resolution $\sim 100 \mathrm{ps}$ for the TOF measurement between TOF0 and TOF1 is needed. All these requirements imply a conservative request of 50-60 ps for single TOF station resolution.

It is not possible to select a single Cerenkov radiator that is sensitive to muons and blind to pions over the entire momentum range. The chosen solution [3] is two different aerogel counters with refractive indices 1.07 and 1.12, each equipped respectively with four 8 " low background EMI 9356KA PMTs from the earlier Chooz experiment.

All the TOF stations share a common design based on fast 1" thick scintillator counters along the X/Y directions (to increase measurement redundancy) read at both ends by R4998 Hamamatsu photomultipliers. All downstream detectors and the TOF1 station needs additional heavy shielding, in addition to mu-metal, against stray magnetic fields. To sustain the high instantaneous incoming particle rate (up to $1.5 \mathrm{MHz}$ for TOF0), active base dividers are used for the TOF PMTs.

The downstream calorimeters (EMCAL) consist of a Pb-scintillating fiber calorimeter (KL), of the KLOE type [4], with 1-mm diameter blue scintillating fibers glued between $0.3 \mathrm{~mm}$ thick grooved lead plates, followed by an electron-muon ranger (EMR), made of a $1 \mathrm{~m}^{3}$ fully sensitive segmented scintillator block. This "spaghetti" design for KL offers the possibility of fine sampling and optimal lateral uniformity. The expected resolution $\sigma_{E} / E \simeq 7 \% / \sqrt{E}$ is fully dominated by sampling fluctuations and the response is linear for electrons or photons in the range 70-300 $\mathrm{MeV}$. The EMR will be made with extruded scintillator bars with WLS fiber readout. In the EMCAL while KL will measure electrons, the EMR will measure precisely the muon range. The TOF0/TOF1/TOF2 and KL detectors have been installed in steps in the MICE Hall in 2008 and 2009, as shown in the right panel of figure 1.

After time-walk corrections and the calibration procedure with impinging beam particles (see reference [5] for details), the TOF detector timing resolution can be measured by using the time difference $\Delta t_{X Y}$ between the vertical and horizontal slabs in the same station (see figure 2). The resolution on the difference is $\sigma_{X Y} \sim 100 \mathrm{ps}$ for TOF0 and TOF2, $\sigma_{X Y} \sim 120 \mathrm{ps}$ for TOF1. ${ }^{1}$

\footnotetext{
${ }^{1}$ This translates into $\sim 50(60) p s$ resolution for the full TOF0/TOF2 (TOF1) detector with crossed horizontal and vertical slabs. The worse resolution of TOF1 is probably due to the poorer quality of the PMTs used.
} 

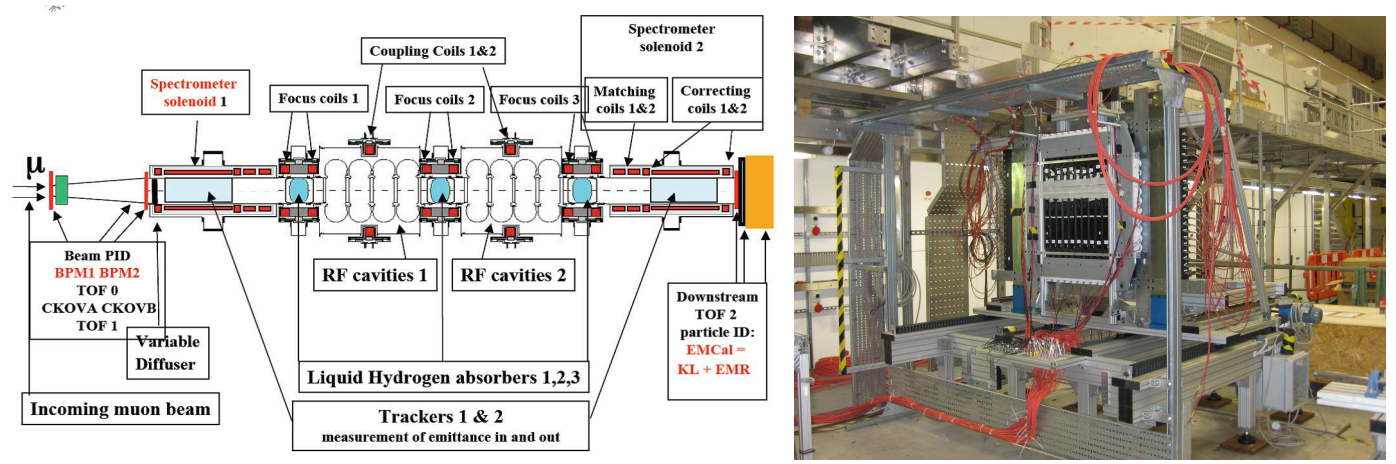

Figure 1: Left panel: layout of the MICE apparatus. Right panel: TOF2 and KL, as presently installed in the MICE Hall.
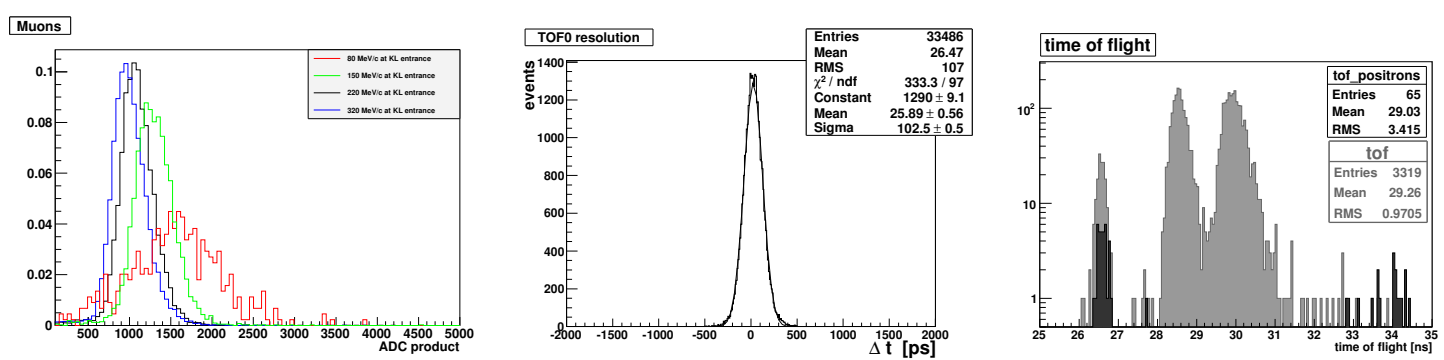

Figure 2: Left panel: $K L$ response to muons. Middle panel: time difference $\Delta t_{X Y}$ between vertical and horizontal slabs in TOF0 (trigger is on TOF1). Right panel: time of flight between TOF0 and TOF1 for the positron (black) and pion (grey) beams in logarithmic scale.

Figure 2 shows, as an example, the distribution of the time-of-flight between TOF0 and TOF1 for a $300 \mathrm{MeV} / \mathrm{c}$ pion beam and a positron beam. ${ }^{2}$ The first peak which is present in both distributions (pion and positron beam) is considered as the time of flight of the positrons and is used to determine the absolute value of the time in TOF1. A natural interpretation of the other two peaks is that they are due to forward flying muons from pion decay and pions themselves. The response of KL to muons with different momenta is also shown in figure 2.

As a conclusion, the first step of MICE, characterizing the incoming $\mu$ beam, has been mainly accomplished and the obtained PID detector performances are compatible with requirements.

\section{References}

[1] A.Blondel et al., MICE proposal, RAL, 2004; Y.Kharadzov, these proceedings.

[2] M.M.Alsharo'a et al., Phys. ReV. ST. Accel. Beams 6,081001 (2003).

[3] L.Cremaldi et al., IEEE Trans. on Nucl. Science 56 (2009) 1475.

[4] A.Aloisio et al., KLOE coll., Nucl. Instr. and Meth. A494 (2002), 326.

[5] R.Bertoni et al., Nucl. Instr. and Meth. A615 (2010)14.

\footnotetext{
${ }^{2}$ This beam is set by starting from the settings for pion beam at $300 \mathrm{MeV} / \mathrm{c}$ and reducing all the currents in the upstream magnets to a nominal $100 \mathrm{MeV} / \mathrm{c}$ momentum. At this momentum only positrons reach the TOF stations.
} 